\title{
X-ray Emission from Comets and Planets in the Solar System
}

\author{
Konrad Dennerl* \\ MPE Garching, Germany \\ E-mail: kodempe.mpg.de
}

Having accompanied X-ray astronomy from its very beginning, solar system X-ray research has experienced a boost during the last two decades. Before 1996, the Sun, Earth, Moon, and Jupiter were the only solar system X-ray sources known. Since then, this number has considerably increased, including now also Mercury, Venus, Mars, Saturn, the Jovian moons Io and Europa, the Io plasma torus, the rings of Saturn, two asteroids, as well as comets as an unexpected new class of X-ray sources, and even the heliosphere itself. The text outlines the sequence of discoveries, describes how the X-ray emissions originate, explains what can be learned from observing them, and concludes with an outlook on future research.

\footnotetext{
Keywords: Comets - Planets - Solar System - X-rays

PACS: 95.30.Cq 95.30.Dr 95.85.Nv 96.12.Jt 96.15.Lb 96.20.-n 96.30.-t 96.30. Cw 96.30.Dz 96.30.Ea 96.30.Gc 96.30.Kf 96.30.L- 96.30.1b 96.30.1d 96.30.Mh 96.30.Pj 96.30.Rm 96.30.Wr 96.30.Ys 96.50.Ci 96.50.Wx
}

Frontier Research in Astrophysics

26 - 31 May, 2014

Mondello (Palermo), Italy

\footnotetext{
* Speaker.
} 


\section{Introduction}

The Sun, Moon, planets and comets have stimulated mankind for millenia, but only a few decades ago it has become possible to observe these objects in X-rays. During the last years this has resulted in surprising discoveries which have consequences exceeding far beyond solar system research. We know now that our solar system contains a wealth of X-ray sources, caused by a rich variety of emission processes. The solar system also offers the unique opportunity in astrophysics to combine remote observations with in-situ measurements. Thus, our solar system can be regarded as a huge laboratory where fundamental physical processes can be studied in detail, processes which also occur at many other locations throughout the Universe.

Due to the large number of discoveries and activities if this field, only a short overview can be given here. The limited space also prevents to give detailed references about individual findings; this information is found in the reviews [1-6] and the references therein. This text is organized as follows: Section 2 will provide a short overview about the history of solar system research and will put it into a more general context. In Section 3 the various physical processes will be described which are important for the X-ray generation in the solar system, and Section 4 will deal with the question what we can learn from studying solar system X-rays. Finally, Section 5 will provide a short outlook to the future.

\section{History and context}

The history of solar system X-ray research can be divided into three periods: an early period (1949-1989), the ROSAT era (1990-1998), and the period from 1999 to present. These periods will be briefly characterized in the following.

\subsection{First period: 1949-1989}

Solar system X-ray research has accompanied X-ray astronomy from its very beginning. Already in September 1949, weak X-ray emission from the solar corona was detected with small Geiger counters aboard a V-2 rocket. Auroral X-rays from the Earth have been observed since the 1960s. The (unsuccessful) attempt to detect X-rays from the Moon in 1962 yielded "evidence for X-rays from sources outside the solar system" and is considered as the beginning of X-ray astronomy. In 1967, fluorescent X-rays from the Earth were first indicated by unexpectedly high background radiation during a daytime stellar X-ray survey by a rocket. Fluorescent X-rays from the Moon were measured in 1971 - 1972 from lunar orbit by Apollo 15 and 16. Jupiter was detected in X-rays in 1979 with the Einstein satellite. So, before 1990 the only solar system objects known to be X-ray sources were the Sun, Moon (detected only from lunar orbit), Earth, and Jupiter.

\subsection{Second period: $1990-1998$}

This is the period where the X-ray satellite ROSAT was operational. The ROSAT era has caused a paradigm change concerning the importance and consequences of solar system X-rays. Already in 1990, ROSAT had made two peculiar observations whose origin and importance, however, were not realized before 1996: (i) ROSAT obtained the first ever X-ray image of the Moon; it exhibited evidence for diffuse $\mathrm{X}$-ray emission which appeared to come from the dark side; 
(ii) when ROSAT was scanning over the sky in order to compose the first all-sky map with an imaging telescope, it observed episodes of overall brightenings of the X-ray sky, called long-term enhancements. These two findings, which originally appeared unrelated, got into context in 1996 with another unexpected ROSAT discovery: X-rays from comets. Only then it became clear that all these phenomena were caused by the same physical process: charge exchange between highly charged ions and neutral gas. While this process had been known long ago and had been studied in detail in atomic physics and even in astrophysics, its astrophysical importance for generating 'standalone' X-rays had been completely overlooked.

\subsection{Third period: 1999 to present}

In the year 1999, two major X-ray observatories were launched: Chandra and XMM-Newton. They provided a major step forward in spatial and spectral resolution as well as in sensitivity. In 2001, the line emission predicted to result from the charge exchange process could be directly observed in the X-ray spectrum of a comet. In 2002, X-rays from Venus and Mars were detected, and additional $\mathrm{X}$-ray sources in the Jupiter system were found: the Galilean satellites Io and Europa and the Io plasma torus. In 2004, X-ray emission from Saturn and its rings was detected, and even an X-ray image of Titan could be obtained, when it passed in front of the Crab supernova remnant. Also in 2004, it was finally proven that the geocorona is emitting X-rays which, together with the X-rays from the heliosphere, do contribute to the soft X-ray background. After 2004, additional X-ray observatories have become available for solar system studies: Swift, launched in 2004, and Suzaku, launched in 2005. In 2011, the solar satellite Hinode succeeded in obtaining X-ray images of a comet which was only $\sim 320000 \mathrm{~km}$ above the solar photosphere.

These remote X-ray observatories have been supplemented by spaceprobes carrying X-ray detectors for observations from close distance, namely NEAR Shoemaker and Hayabusa, which investigated the surface chemistry of two asteroids by X-ray spectroscopy of fluorescently scattered solar X-rays in 2000 and 2005. Since 2011 this technique is also applied by the Messenger spaceprobe in orbit around Mercury for mapping major elemental abundances on its surface. For the Moon, the early X-ray fluorescence measurements pioneered by Apollo 15 and 16 were complemented by SMART-1 (2003-2006), Kaguya (2007-2009), and Chandrayaan-1 (2008-2009) from lunar orbit.

\section{Physical processes}

The origin of all the currently known X-ray emission in the solar system can be traced back to three basic ways by which the necessary energy is supplied: by heat, by velocity, and by charge. This will be briefly described in the following.

\subsection{Heat: thermal emission}

The X-ray emission of the Sun is generated in the hot solar corona. With temperatures of several million degrees, this is the only location in the solar system which is capable of emitting thermal X-rays, with one remarkable exception: the X-ray emission from a comet (C/2011 W3) which was observed when it was only $\sim 320000 \mathrm{~km}$ above the solar photosphere, was suggested 
to arise directly from the ionization of cometary material. At larger distances, the temperatures of solar system bodies are much too low to produce thermal X-rays. Nevertheless, thermal emission can be an important component of their X-ray radiation: whenever the X-rays emitted by the hot solar corona hit a target of sufficient density, they will be scattered, either elastically or fluorescently. This happens at the atmospheres or surfaces of planets (including their moons and rings) and on small bodies (asteroids and cometary nuclei). Scattered solar X-rays have been observed from the atmospheres of Venus, Earth, Mars, Jupiter and Saturn and from the surfaces of Mercury, our Moon, and two asteroids. Also the X-ray emission observed from the rings of Saturn was suggested to be (in part) caused by scattering of solar X-rays on an 'atmosphere' above the rings. Interestingly, scattered solar X-rays have not been observed from the exospheres of planets, from cometary gas or from gas in the heliosphere. This is caused by the fact that, due to the low cross section of X-ray scattering, tenuous gas does not provide enough targets for making this process efficient.

\subsection{Velocity: energetic electrons and ions}

A very common process for powering X-rays throughout the Universe is by the kinetic energy of particles. When charged particles of sufficient velocity are deflected, they emit X-rays by bremsstrahlung. This is the case for energetic solar wind electrons interacting with planetary magnetospheres, causing the auroral X-ray emission of Earth and Jupiter. The magnetic field of Jupiter is so strong that it can even accelerate non-energetic solar wind electrons to the required energies. Such electrons are thought to be also responsible for the $\mathrm{X}$-rays observed from the Io plasma torus. In addition to electron bremsstrahlung (which produces an X-ray continuum), electrons can generate $\mathrm{X}$-rays by collisional ionization (producing characteristic $\mathrm{X}$-ray line emission), as observed in the polar regions of the Earth and the night side of Mercury. The strong magnetic field of Jupiter is also capable of accelerating ions to the energy required for producing X-rays by bremsstrahlung and collisional ionization. This process was suggested to contribute to the $\mathrm{X}$-ray emission of Jupiter and to be responsible for the X-ray emission of its moons Io and Europa.

\subsection{Charge: highly charged ions}

This is the 'youngest' process: despite having been investigated in great detail for many decades, the importance of charge exchange for X-ray astrophysics was only realized in 1996, i.e., 34 years after the beginning of $\mathrm{X}$-ray astronomy, with the discovery of cometary X-rays. This process is fundamentally different from those typically studied in X-ray astrophysics, which involve energetic electrons, because here the $\mathrm{X}$-rays are not powered by kinetic energy but by the energy which is stored in highly charged ions. This energy is released whenever the ions recombine with electrons, like in comets when the solar wind encounters their extended atmospheres. As this process is characterized by very large cross sections $\left(\sim 10^{-15} \mathrm{~cm}^{2}\right)$, charge exchange induced $\mathrm{X}$-rays can be utilized as a highly sensitive sensor for tenuous amounts of gas. This could not only be demonstrated on comets but also on Mars, where a novel method for remote global studies of planetary outgassing was pioneered. Due to the high cross section, also the exosphere of Earth and even the heliosphere are X-ray bright. Their diffuse glow is superimposed on the radiation which we observe from celestial targets. Thus, any $\mathrm{X}$-ray observation made from within the solar system may be affected by charge exchange. 


\section{What do we learn?}

The X-rays which we receive from comets and planets contain a wealth of information which can be utilized in various ways. This will be shown in the following, first for our own solar system and for other stellar systems, and then for physics and astrophysics in general.

\subsection{Solar System}

$\mathrm{X}$-ray observations of comets and planets provide us a complementary view of otherwise familiar objects. By X-ray observations of comets, we do not only learn about these objects, but get also valuable information about the solar wind, while $\mathrm{X}$-ray observations of planets reveal properties which are difficult to obtain by other techniques.

\subsubsection{Comets and the Solar Wind}

An important property of comets are their gas and dust production rates, which cannot be easily determined separately. X-ray observations, however, make it possible to measure the pure gas production rate, unaffected by dust, as the $\mathrm{X}$-ray emission is the result of charge exchange with gas. At the same time, the X-rays contain information about the solar wind, a stream of particles emitted from the Sun with a typical velocity of $\sim 400 \mathrm{~km} \mathrm{~s}^{-1}$. After having become ionized in the hot solar corona, the rapid expansion of the plasma prevents the ions from recombining with electrons. However, when the ions encounter the gas cloud around a comet or planet, they find a reservoir of electrons, and recombination can take place. For highly charged heavy ions this process is accompanied by $\mathrm{X}$-ray line emission with characteristic spectral signatures. Thus, the $\mathrm{X}$-ray spectra of comets contain information about the heavy ion content in the solar wind and its ionization stage, information which would otherwise only be accessible by in-situ measurements.

In this way, comets can be utilized as natural space probes for measuring the ionization state and chemical composition of the solar wind. As the orbits of comets are not confined to the ecliptic plane, this can be done at various heliographic latitudes, and also at various phases in the solar cycle. Such measurements are not only important for studies of our Sun, but also of solar-like stars, which exhibit similar activity cycles. While the activity cycles of such stars can be directly observed, this is not possible for the heavy ion content of the stellar winds. In this context it is useful to get such data for the solar wind as a reference, from X-ray observations of comets.

\subsubsection{Planets with atmospheres}

Due to the high cross sections for charge exchange, $\mathrm{X}$-ray observations open a novel window for global, remote imaging of planetary exospheres and their response to solar activity. This is particularly the case for Venus and Mars, which do not possess any major global magnetic field, so that the solar wind can directly interact with exospheric gas. At Mars, exospheric X-ray emission could already be traced out to eight planetary radii. Thus, X-ray observations are a powerful tool for studying planetary outgassing. Such studies are important for our understanding of the evolution of planetary atmospheres and the evolution of life. While X-rays caused by charge exchange reveal details of the outer exospheres, scattered solar X-rays sample the upper atmospheric layers: at Venus and Mars, scattering is most efficient at heights of $110-140 \mathrm{~km}$, giving us observational access to regions well above the uppermost clouds. 
At Jupiter, the X-rays resulting from bremsstrahlung, collisional ionization, and charge exchange provide a window into the highly complex interactions between the solar wind, its magnetosphere, its moons, and the Io plasma torus. At Saturn, many questions are still open, like the precise origin of the $\mathrm{X}$-ray emission from its rings. Due to the large distance from the Sun and Earth, the X-ray signal from Saturn is very low. This is even more the case for Uranus and Neptune, where no X-ray emission has been detected yet. A major progress in studying the X-ray properties of the outer planets could probably be achieved by in-situ measurements with spaceprobes equipped with X-ray detectors.

\subsubsection{Mercury, Moon, Asteroids}

For solar system bodies with no atmosphere, the chemical composition of their surface can be deduced from spatially resolved X-ray spectra, utilizing scattered solar X-rays. This is now applied as a standard technique for Mercury, our Moon, and asteroids. Although neither the Moon nor Mercury do possess an atmosphere, they are surrounded by a very tenuous exosphere, which can be studied by charge exchange induced X-rays. An X-ray detection of the lunar exosphere has recently been reported. For Mercury, which is too close to the Sun to be observed in X-rays from the Earth environment, it is planned to perform such studies with the spaceprobe BepiColombo.

\subsection{Physics and Astrophysics}

There is a remarkably close link between solar system X-ray studies on the one hand, and physics and astrophysics on the other hand. This is in particular true for the charge exchange process.

\subsubsection{Comets}

Comets are ideal targets for studying the physics of charge exchange, because their X-ray emission is the direct result of charge exchange reactions, unaffected by electron bremsstrahlung or scattered solar X-rays. A detailed understanding of this fundamental process is an essential requirement for plasma diagnostics in general, which is an important tool for astrophysics as well as for applied physics, like nuclear fusion. Despite its apparent simplicity, the derivation of detailed parameters, like the state selective velocity dependent cross sections, is a most challenging task, both theoretically and experimentally. In this context, comets represent an excellent laboratory with a clean experimental setup, where highly charged ions interact with cold neutrals at low density, far away from thermal equilibrium, and this 'experiment' can be investigated with the full range of currently available $\mathrm{X}$-ray instrumentation. Thus, $\mathrm{X}$-ray observations of comets can be considered as benchmarking experiments for testing our understanding of the physics of charge exchange.

\subsubsection{Various Astrophysical Environments}

Charge exchange induced $\mathrm{X}$-ray emission in the geocorona and heliosphere is now realized to represent an additional X-ray "background" component, by which any X-ray observation made from within the solar system may be affected. This knowledge is important for a correct interpretation of X-ray observations. Otherwise this diffuse X-ray emission could be, e.g., misinterpreted as evidence for extended $\mathrm{X}$-ray haloes around nearby galaxies. The insight that part of the observed 
$\mathrm{X}$-ray emission may be of solar system origin has already required to revise the models describing the properties of the Local Hot Bubble.

Charge exchange induced X-ray emission, however, is not confined to our solar system; it will occur wherever highly ionized plasma interacts with neutral or less ionized gas. Such scenarios are found throughout the Universe, like in supernova remnants and star forming regions in our galaxy and beyond. Indications for this process have already been found to occur in clusters of galaxies. The presence of such an emission component in galaxy clusters might even have cosmological implications, because it may mimic thermal plasma emission of higher temperature and thus lead to an overestimation of the amount of Dark Matter.

\section{Outlook}

Over the last decades, solar system X-ray observations have revealed that even our immediate astronomial environment is full of surprising phenomena that are awaiting discovery. With future $\mathrm{X}$-ray missions, this trend is likely to continue. A very promising mission will be Astro- $\mathrm{H}$, scheduled to be launched in 2016, the first space mission which will perform X-ray observations with a microcalorimeter, providing unprecedented spectral non-dispersive resolution. In 2017, the X-ray satellite eROSITA will be in the process of performing a total of eight all-sky surveys over the period of four years. This will be the first satellite to observe the X-ray sky from a halo orbit around the Sun-Earth L2 point, sufficiently far away from Earth to be unaffected by charge exchange in the geocorona. Also in 2017, the space probe BepiColombo will be launched to Mercury, which it will reach in 2024. It carries an imaging X-ray spectrometer which will be used for pioneering studies of charge exchange induced X-rays from Mercury's exosphere. Further in the future, in 2028, a major X-ray observatory, Athena, is scheduled for launch. Like eROSITA, Athena will observe the X-ray sky from a halo orbit around L2. With its unparalleled sensitivity and spectral resolution, Athena is likely to provide a quantum leap in many areas of astrophysics, including the study of solar system objects.

Having accompanied X-ray astronomy from its very beginning, $\mathrm{X}$-ray studies of solar system objects have experienced a boost during the last decades, with implications reaching far beyond solar system physics. In particular the importance of the process of charge exchange for the generation of X-rays throughout the Universe is getting increased attention and has already led to a paradigm change in some areas. In 2012, a whole special issue of Astronomical Notes was devoted to charge exchange, illustrating that this topic can be regarded as a new frontier in Astrophysics.

\section{References}

[1] Lisse, C. M., Cravens, T. E., Dennerl, K.: 2004, Comets II, Eds. Festou, M. C., Keller, H. U., Weaver, H. A., 631

[2] Krasnopolsky, V. A., Greenwood, J. B., Stancil, P. C.: 2004, Space Science Reviews 113, 271

[3] Bhardwaj, A., et al.: 2007, Planetary and Space Science 55, 1135.

[4] Dennerl, K.: 2010, Space Science Reviews 157, 57.

[5] Dennerl, K., et al.: 2012, AN 333, 324. 
[6] Bhardwaj, A., Lisse, C. M., Dennerl, K.: 2014, Encyclopedia of the Solar System, 3rd Edition, Chapter 48, Eds. Spohn, Breuer, Johnson, Elsevier, ISBN 9780124158450

\section{DISCUSSION}

DMITRY BISIKALO: Could you explain the symmetric shape of the $\mathrm{X}$-ray region in comets while the "optic" shape is asymmetric?

KONRAD DENNERL: The optical appearance of a comet is dominated by its dust while the $\mathrm{X}$-ray emission is determined by its gas content. The dust particles are initially emitted mainly sunwards, but get then slowly deflected by solar radiation, giving the comet its characteristic optical shape, with a head and a tail. As the velocity of the dust in the tail relative to the comet is low, the dust tail appears often broad and curved, depending on the specific viewing geometry. The situation is considerably different for the gas: the gas molecules and atoms are fairly immune against solar radiation pressure, but can get ionized by UV radiation. As soon as this happens, they are quickly swept away by the solar wind with a velocity of a few hundred $\mathrm{km} \mathrm{s}^{-1}$, forming a straight plasma tail. Before that, they are moving radially away with $\sim 1 \mathrm{~km} \mathrm{~s}^{-1}$. As the ionization lifetime at $1 \mathrm{AU}$ distance from the Sun is about $10^{6} \mathrm{~s}$, a huge approximately spherical neutral gas coma with a radius of $\sim 10^{6} \mathrm{~km}$ develops, which can be observed in Ly- $\alpha$. The neutral gas coma provides a reservoir of electrons which is used by the highly charged solar wind ions for recombination, thereby emitting $\mathrm{X}$-rays. The observed crescent shaped $\mathrm{X}$-ray emission which is shifted sunward and which is symmetric with respect to the Comet-Sun axis can be well reproduced by the simplifying assumptions that a parallel stream of ions penetrates a collisionally thick gas cloud with a $r^{-2}$ density profile.

SOLEN BALMAN: What is the origin of the $\mathrm{X}$-ray emission associated with the rings of Saturn?

KONRAD DENNERL: The originally favoured explanation was that they are caused by fluorescent scattering of solar X-rays, motivated by the fact that the Cassini spaceprobe had found evidence for a tenuous oxygen atmosphere around the icy rings. This explanation was consistent with the observed X-ray flux and X-ray spectrum, which is essentially confined to the $\sim 0.47-0.62 \mathrm{keV}$ region and identified as $\mathrm{O}-\mathrm{K}_{\alpha}$ emission. Later observations, however, have indicated that the strength of the X-ray emission did not decrease as expected when the solar activity cycle evolved to its minimum, and it the presence of an alternative, perhaps additional, process was suggested: lightning-induced electron beams, which may be the origin of the spokes observed in the Saturnian rings, may also energise the icy particles in the rings and cause them to emit fluorescent $\mathrm{O}-\mathrm{K}_{\alpha}$ $\mathrm{X}$-rays. Detailed studies of the $\mathrm{X}$-ray emission are hampered by low statistics: there is only one $\mathrm{X}$-ray photon emitted from the rings for every $10^{12}$ visible photons. 